\title{
EXTERNALLY POWERED DIELECTRIC LOADED WAVEGUIDES AS ACCELERATING STRUCTURES
}

\author{
Wei Gai, R. Konecny and J. Simpson \\ Argonne National Laboratory, Argonne, IL 6039, USA
}

\begin{abstract}
There have been numerous studies examining the use of dielectric materials in accelerating structures in the past. Such studies and related experiments generally concluded that dielectric losses, with the resulting high power requirements, and field breakdown problems made such devices not very attractive. However, development of high dielectric constant, low loss materials plus new demands for emittance preserving acceleration warrant a new look at the idea. In this paper, we discuss reference parameter designs for S-band and X-band structures and show that dielectric loaded guide may now be practical and have certain advantages over conventional iris loaded structures. Preliminary experimental test results of Xband dielectric devices are reported.
\end{abstract}

\section{INTRODUCTION}

The proposed use of rf driven dielectric based structures for particle acceleration can be traced to the early 50's [1]. Since then, numerous studies have examined the use of dielectric materials in accelerating structures. Such studies and related experiments generally concluded that high dielectric losses with the consequent high power requirements, and field breakdown problems made such devices unattractive compared with conventional copper cavity. However, recent development of high dielectric constant $(\varepsilon \sim 20-40)$, low loss materials $(\mathrm{Q} \sim 10,000$ $40,000)$ [2] plus new demands for emittance preserving acceleration warrant a new look at the idea [3,4].

Some potential advantages of the dielectric loaded structures are:

- Simplicity of fabrication - the device is little more than a tube of dielectric surrounded by a conducting wall cylinder. This can be a great advantage for high frequency $(10 \mathrm{Ghz})$ structures compared to conventional structures. Also the relatively small diameter of these devices facilitates placement of quadrupoles around the structures.

- Reduced single bunch beam break-up (BBU) - the lowest frequency deflecting mode is almost always lower than that of the acceleration mode.

- Simple reduction of coupled bunch - bunch effects it has been shown that it is relatively straightforward to build deflection damping into dielectric structure so that very large absorption (I. e. $250 \mathrm{~dB} / \mathrm{m}$ ) of all but $\mathrm{TM}_{0 \mathrm{n}}$ modes can be obtained [5].

Potential problems for dielectric loaded structures are charge accumulation from beam halo, surface breakdown and thermal heating. Whether these problems are fatal or not can only be answered through experiments.

In this paper, we discuss reference parameter sets for both S- and X-band structures and show that dielectric loaded devices may now be practical and have certain advantages over conventional iris loaded structures. Preliminary experimental test results of X-band dielectric devices are reported here in section 5.

\section{A TRAVELLING WAVE DIELECTRIC LOADED ACCELERATING STRUCTURE}

The dielectric travelling wave accelerator has simple geometry. Considering a cylindrical structure partially filled with dielectric material $(\varepsilon)$ with inner radius a, outer radius $\mathrm{b}$ and conducting wall on the outside. There are also two ports on the side for RF coupling purposes, as shown in Figure 1. The axial electric fields inside the structure can be solved for exactly as

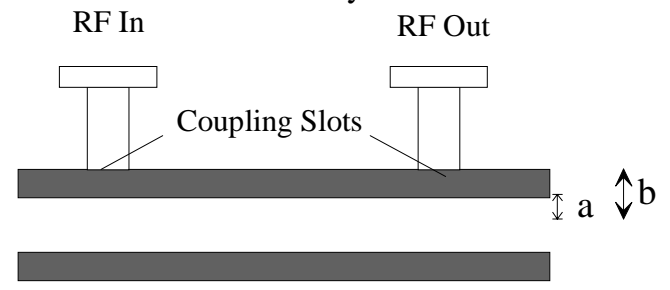

Figure 1. Schematic diagram of a dielelectric loaded travelling wave accelerator.

$$
\begin{aligned}
& E_{z}{ }^{(1)}(\omega, r, z, t)=E_{0} I_{0}(k r) e^{i(k z z-\omega t)} \\
& E_{z}{ }^{(2)}(\omega, r, z, t)=\left[B_{1} J_{0}\left(s_{1} r\right)+D_{1} N\left(s_{1} r\right)\right] e^{i\left(k_{z} z-\omega t\right)}
\end{aligned}
$$

Here $E_{0}, B_{0}$ and $D_{1}$ are the field amplitudes in the region 0 (vacuum) and 1 (dielectric) respectively and are related by boundary conditions, and

$$
\begin{aligned}
& k^{2}=\frac{\omega^{2}}{c^{2}}\left(1-\beta^{2}\right) \\
& s^{2}=\frac{\omega^{2}}{v^{2}}\left(\beta^{2} \varepsilon-1\right)
\end{aligned}
$$

where $\beta c=v=\omega / k$ is phase velocity of the wave travelling inside the tube. Therefore $\beta$ determines the synchronism of the wave and the accelerated particles. By properly choosing $a, b$ and $\varepsilon$, one can adjust phase velocity accordingly. Thus this proposed scheme works not only for electron acceleration which typically has high phase velocity, but also for low phase velocity 
particle acceleration, such as required for heavy ions. The transverse electric field can be written as

$$
E_{r}=\frac{i}{\frac{\omega}{v}\left(\beta^{2} \varepsilon-1\right)} \frac{\partial E_{z}}{\partial r}
$$

and the magnetic field $H_{\phi}=\varepsilon E_{r}$ everywhere inside the tube. By matching the boundary conditions at $a$ and $b$ ( $E_{z}$ and $D_{r}$ continous), all the fields components can be calculated accordingly.

The stored energy per unit length $U$ in the tube is the sum of contributions from both vacuum and dielectric regions, and can be expressed as

$$
\begin{aligned}
U & =U_{\text {in }}+U_{\text {out }} \\
& =\sum_{0,1} \pi \int\left(\varepsilon \varepsilon_{0} E^{2}+\mu_{0} B^{2}\right) r d r \\
& =E_{0}{ }^{2} u
\end{aligned}
$$

where $u$ is a geometric factor which solely depends on the structure geometry and dielectric constant. For a given RF power, the axial electric field in the center region of the tube can be expressed as

$E_{0}=\left[\frac{P}{u \beta_{g} c}\right]^{\frac{1}{2}}$

where $v_{g}$ is the group velocity. The dielectric loss per unit length is then found from:

$\eta=\frac{2 \pi \delta f U_{\text {out }}}{v_{g}\left(U_{o u t}+U_{i n}\right)}$

The electric fields in the vacuum region described by equation 1 and 3 have very interesting chraracteristics. When $k \rightarrow 0$, i.e., the phase velocity of the wave is c, $E_{z}$ is constant in $\mathrm{r}$. This implies that there are no focusing and de-focusing forces for a relativistics particle travelling inside the vacuum chamber. This is critical for emittance preserving in the Linear Accelerator, particularly for the high brightness electron gun development.

\section{DESIGN FOR S- AND X- BAND LINAC STRUCTURES}

There were proposed schemes using S-Band dielectric structures in the last several years $[3,4]$. In the past, the dielectric materials available have the characteristics of relatively small $\varepsilon(<10)$ and relative high loss tangent $(\delta>$ 10-4). However, recently dielectric materials with $\varepsilon>20$, $\delta<10-4$ and with DC breakdown voltage $>10 \mathrm{MV} / \mathrm{m}$ have become commercially available [2]. In this section, we show several design parameters with different dielectric constants and loss tangents. We compare our design results with SLAC S-band and NLC X-band conventiona cavity designs. Although both SLAC and NLC designs were for constant gradients, we could certainly taper the dielectric tubes to achieve a constant gradient acceleration structure. But we believe that a constant impedance structure would show the same characteristics as constant gradient and a fair comparison can be made.

\section{a. $S$ band structures}

We have developed a self consistant computer code to calculated the properties of the dielectric travelling wave structures. The following table summarises the results for two possible dielectric S-band structures. As shown in this table, the group velocity is much higher than SLAC cavity although HEM11 is lower.

Table I: DLG Reference Design for S - Band

$\begin{array}{lll}\text { Scheme } & \text { I } & \text { II } \\ \text { Material } & \text { MgCaTi } & \text { BaTi } \\ \varepsilon \text { (diele. const.) } & 20 & 35 \\ \text { Loss tangent } \delta & 10^{-4} & 3 \times 10^{-5} \\ \text { Inner Radius a } & 0.5 \mathrm{~cm} & 0.5 \mathrm{~cm} \\ \text { Outer Radius b } & 1.20 \mathrm{~cm} & 1.09 \mathrm{~cm} \\ \text { HEM11 } & 2.304 \mathrm{GHz} & 2.71 \mathrm{GHz} \\ \text { Shunt Imped. } & 10 \mathrm{M} \Omega / \mathrm{m} & 7.4 \mathrm{M} \Omega / \mathrm{m} \\ \text { group velocity } & 0.05 \mathrm{c} & 0.028 \mathrm{c} \\ \text { Attenuation } & 0.6 \mathrm{~dB} / \mathrm{m} & 0.36 \mathrm{~dB} / \mathrm{m} \\ \text { Power } & \text { required } & 13.6 \mathrm{MW} \\ \text { (10MV/m) } & & \end{array}$

\section{b. $11.4 \mathrm{GHz}$ structures}

We have also considered two possible designs for X-band structure $(11.4 \mathrm{Ghz})$. Unlike the case in S-band, the group velocity for the NLC design is in the range from $0.03 \mathrm{c} \sim$ $0.04 \mathrm{c}$ [5]. Thus dielectric loaded structure is having a comparable shunt impedence and group velocity as indicated in the table below.

Table II: DLG Reference Design for X - Band

$\begin{array}{lll}\text { Material } & \text { MgCaTi } & \text { BaTi } \\ \varepsilon \text { (diele. const.) } & 20 & 35 \\ \text { Loss tangent } \delta & 10^{-4} & 3 \times 10^{-5} \\ \text { Inner Radius a } & 0.3 \mathrm{~cm} & 0.3 \mathrm{~cm} \\ \text { Outer Radius b } & 0.456 \mathrm{~cm} & 0.416 \mathrm{~cm} \\ \text { HEM11 } & 9.96 \mathrm{~Hz} & 10.2 \mathrm{GHz} \\ \text { Shunt Imped. } & 37 \mathrm{M} \Omega / \mathrm{m} & 47 \mathrm{M} \Omega / \mathrm{m} \\ \text { group velocity } & 0.057 \mathrm{c} & 0.035 \mathrm{c} \\ \text { Attenuation } & 1.2 \mathrm{~dB} / \mathrm{m} & 1.1 \mathrm{~dB} / \mathrm{m} \\ \text { Power required } & 2.73 \mathrm{MW} & 2.02 \mathrm{MW}\end{array}$

$(10 \mathrm{MV} / \mathrm{m})$

As shown above, one of the common characteristics of all the structures is that the frequency of HEM11 (first deflection mode) is lower than that of the acceleration modes. Because the deflection force is a function of $\sin (\mathrm{kz})$, this implies a very different and improved conditions for single bunch BBU problem compared to conventional structures where the HEM11 is always a higher in frequecy than the acceleration mode TM01. 


\section{DAMPENING HIGHER ORDER MODES IN THE DIELECTRIC STRUCTURES}

Unlike conventional disk loaded accelerator, non axial symmetric mode dampening can be achieved easily by cutting some longitudinal slots in the conducting wall. The deflection modes are non-axisymmetric hybrids containing both axial electric and magnetic fields. These hybrid modes are comprised of all six cylindrical field components and will require both azimuthal and axial surface currents on the outer conductor. If the outer conductor is segmented to allow only axial surface currents, the deflection modes will not be confined and will be radiated beyond the outer wall. If this outer region is filled with rf absorbing material, the deflection modes will be highly attenuated. Test on the bench and with electron beam at AATF [6] have shown that 200 $\mathrm{dB} / \mathrm{m}$ attentuation can be achieved easily.

\section{CONSTRUCTION AND TEST OF AN $11.4 \mathrm{GHZ}$ PROTOTYPE DIELECTRIC STRUCTURES}

We have constructed a $11.4 \mathrm{GHz}$ dielectric loaded travelling wave accelerating structure using dielectric from TransTech, with dimensions of $a=2.9 \mathrm{~mm}, b=$ $4.59 \mathrm{~mm}$ and dielectric constant of 20 . The length of the structure is $10 \mathrm{~cm}$. This is a comparable structure as in the Type I of the Table 2.

It is also important to emphasize that efficient $\mathrm{rf}$ coupling to dielectric structures is required for wakefield step-up transformers. The results obtained here are directly applicable to the optimized design of the coupled wake tube device experiments planned for the AWA [6].

The RF coupling scheme we used here is similiar to conventional disk-washer RF cavities (Side coupled). Impedance matching of the coupling slotsis more difficult in the high $\varepsilon$ dielectric case because the outer radius of the dielectric tube is much smaller than the waveguide. By careful adjustment of the coupling slots and monitoring the S-parameters using an HP8510C network analyzer, we have achieved reflection $\mathrm{S} 11<-28 \mathrm{~dB}$ and $\mathrm{S} 21>-1.8 \mathrm{~dB} @ 11.45 \mathrm{Ghz}$ as shown in the Figure 2 and 3 respectively.

We have also tested a standing wave dielectric structure with an RF side coupled port. We achieved following S11<-30 dB @ 11.39 Ghz. The loaded Q from this measurement is 5000, which is in agreement with the manufacturer's specification.

We plan to continue engineering studies of this accelerating structure with improved RF coupling and mechanical fixture to allow operation in vacuum. With $100 \mathrm{MW}$ power, we can test this structure at $60 \mathrm{MV} / \mathrm{m}$ gradient. We are planning to perform the high power tests using the X-band rf system at SLAC/NLCTA.

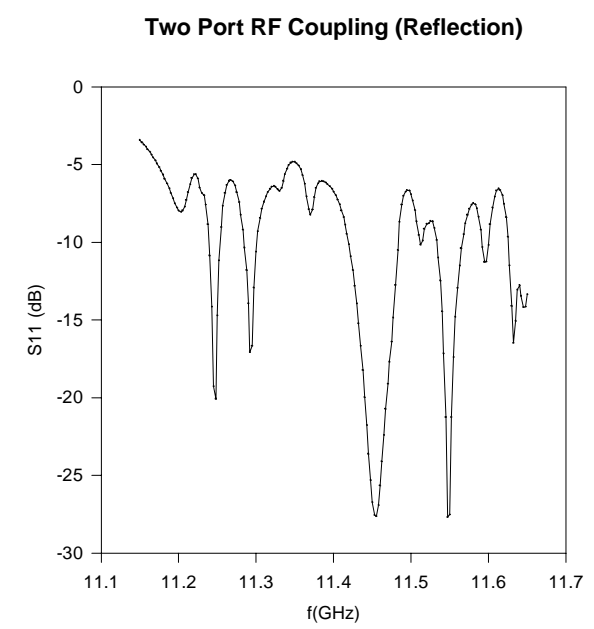

Figure 2. S11 of the optimally coupled waveguide results.

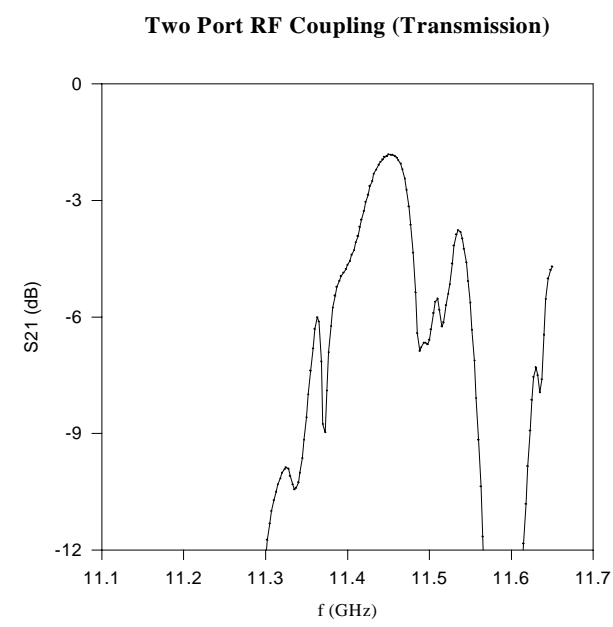

Figure 3. S21 for the optimally coupled wave

In summary, we have studied the dielectric loaded waveguide as a viable alternative for the accelerating structures. We have shown that this structure can be competitive, particularly with the higher frequency demand for the future high energy machine. Some pratical issue can only be answered through careful experimental studies and new materials development. This work is supported by DOE, High Energy Physics Division under contract No. W-31-109-ENG-38.

\section{REFERENCES}

[1] G. Flesher and G. Cohn, AIEE Transactions, 70, 887 (1951)

[2] Trans-Tech., Catalog, 5520 Adamstown, Adamstow, MD 21710

[3] J. Simpson, ANLHEP-WF 174, (1992)

[4] T. B. Zhang et. al., in Proceedings of 7th Advanced Accelerator Worksop, (1996).

[5] SLAC repor 474, 1996

[6] E. Chojnacki et al, J. Appl. Phys. 69,6257 (1991) 